\title{
The design and analysis of the mechanical property test platform frame for marine structures
}

\author{
Tang Wen-xian ${ }^{1}$, Li Cun-jun ${ }^{2}$, Su Shi-jie ${ }^{1}$, WangBo ${ }^{1}$ and Wang Hai-rong ${ }^{2}$ \\ 1 Jiangsu University of Science and Technology, College of Mechanical Engineering, China \\ ${ }^{2}$ Zhoushan Institute of Calibration and Testing for Quality and Technology Supervision, China
}

\begin{abstract}
In order to realize mechanical properties test of the emergency towing arrangements, anchor chain and other large marine components, a large-tonnage and multi-function horizontal tension test platform is put forward in this paper. The paper analyzes the disadvantages of the frame structure of the existing experimental platform and designs the composite structure of the concrete and steel. The steel has good elasticity and toughness, the concrete has good performance of high rigidity and compression resistance, so the composite structure takes the advantages of both. The sharp of the frame is like "L", which can be used to testing different projects. The paper gives the detailed size and analysis procedure of the frame. And it finds a more reasonable structure by means of finite element to analyze and optimize. The frame structure has the advantages of low weight and cost. The platform has been installed in Zhoushan, China successfully.
\end{abstract}

Keywords: mechanical property test platform, frame design, analyze and optimize

\section{Introduction}

Marine structures include: Emergency towing device; Chain; Wire rope, such as emergency steel towline; Steel members, such as thin rod, triangle plate, etc 1. Their performance is closely related to the safety of marine transportation. As a result, most of countries formulate the corresponding testing standards to ensure that the performance of marine structures meets the requirements of ocean transportation. There are many test items, so it is not economical and takes up too much space to design dedicated testing institutions for different marine structures. The emergence of comprehensive test platform has solve above problems successfully and play a key role for the performance testing and the control of quality.

The test platform structure is mainly divided into the vertical and the horizontal. Vertical test platform takes up little space and was used in small size tensile specimen generally. Large size parts whose longitudinal length is generally greater than $3 \mathrm{~m}$, such as hook assembly, cable, wire rope, need to adopt horizontal structure test platform instead of vertical 2. For super-long and extra-wide largescale horizontal tensile test platform, the traditional frame of structure is popularly processed by box structure of the steel plate or by the steel plate directly. Traditional structure not only has the disadvantages of great weight and huge volume, but also lacks of enough rigidity and takes a lot of money. In order to achieve the test platform of mechanical properties for a variety of marine components, we need a frame that has a wide range of application, high reliability, high precision, simple structure and is easy to implement. The frame is used for install and fixes each test equipment, which makes the whole test of marine structures completed successfully.

The platform the paper mentioned uses composite structure of steel and concrete on a very large test platform at the first time, which solves the problems of compatibility of deformation between two kinds of materials. It takes the advantages of the high tensile strength of steel structure and is combined with the rigid of concrete. During the experiments , axial tensile stress and impulse load are borne by the platform structure, and the part of concrete is under pressure only. The concrete is mainly used for enhance longitudinal stiffness of the platform. By the calculation, the test platform reduces $70 \%$ of the steel usage compared with traditional frame structure, which greatly saves the manufacturing cost at the same time.

\section{Main technical parameters of the platform}

The platform is multifunctional mechanical properties testing equipment. The test object is large marine components .The platform can undertake strength test of

\footnotetext{
${ }^{\mathrm{a}}$ This work was financially supported by public welfare industry research foundation of General Administration of Quality Supervision Inspection and Quarantine of the People's Republic of China (Grant No.201310111)
} 
emergency towing device, as well as other main components of emergency towing unit, such as chafing chain, towing, stopper, which reduces the risk of ocean transportation greatly. The test load requirements of the object for the platform is as follows: a. emergency towing device: $2000 \mathrm{KN} \sim 4000 \mathrm{KN}$; b. other marine steel members satisfy the requirement of $120 \mathrm{KN} \sim 12000 \mathrm{KN}$. In order to meet the test requirements of the emergency towing steel wire ( around $90^{\circ} /$ down $30^{\circ}$ ), the shape of structure is designed as approximate $\mathrm{L}$, which can be used for tests of different projects. $3 \mathrm{~d}$ model of the platform is shown in figure 1 .

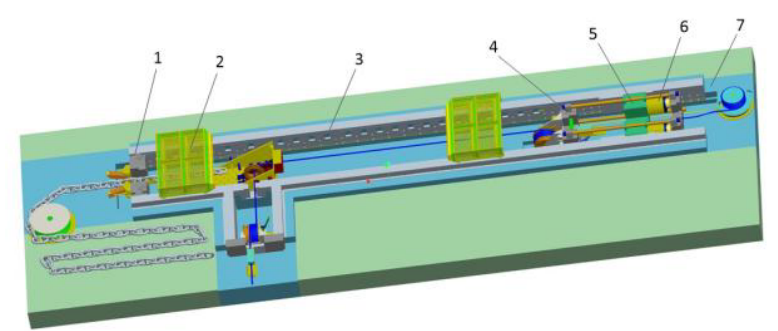

1-Fixed beam, 2 - defend net, 3 - the frame, 4 walking beam, 5 - oil cylinder, 6 - cylinder, 7 concrete

Figure 1. 3d model of the platform

\section{Design of the frame}

Traditional large tensile test platform is usually composed of boxes. The boxes are manufactured respectively and connected with each other by large bolts. The advantage of the form is widely used in large test platform with mature technology both at home and abroad. Shortage is that the weight of the individual components are great and the cost is high in manufacturing, transportation and installation. Besides, it is easy to appear larger stress in the place of bolt, which is very adverse to the strength of the whole structure.

The study of applying the reinforced concrete structure to manufacture the frame abroad began in the 1950s has achieved certain success. But the structure used in large horizontal tensile testing platform is reports rarely. In our country, since the 1960 s, several units has studied and built the platform of reinforced concrete structure of hydraulic press, which provides a new train of thought to the design of the frame of the test platform 3. The structure of the frame has the advantages as follows: a. metal materials can be saved about 50\% $70 \%$ compared with steel type; b. reduce costs. Due to the large castings and forgings as well as the manufacture process is expensive, the structure can save cost about $50 \% \sim 70 \%$; c. reinforced concrete structure can cast and operating conveniently on the spot. And the manufacturing cycle can be shortened by more than half.

Of course, reinforced concrete structure also exists disadvantages: the technology requirement of concrete construction is high; the structure has large volume and cannot tear down and remove, etc. Therefore, reinforced concrete structures only apply to the fixed place.
The paper designs the frame of steel-concrete composite structure as shown in figure 2. The frame adopts the semi-submersible structure with the length of $33.25 \mathrm{~m}$ and the width of $7.83 \mathrm{~m}$. The structure is mainly composed of several segments of Q345B steel plates whose cross section are $1250 \mathrm{~mm} * 120 \mathrm{~mm}$ and punch two pairs of slotted holes every $900 \mathrm{~mm}$. The frame fixes in C30 concrete which is casted as a whole through foundation bolts of M24 x $500 \mathrm{~mm}$. The upper of the frame is composed of welding rectangular box made by steel plate of $16 \mathrm{~mm}$ thickness. Q345B steel plate has good elasticity and toughness, so repeated tension and compression within the elastic range do not lead to permanent deformation or damage of the frame structure. At the same time, concrete structure has good performance of high rigidity and compression resistance, but the tensile and impact resistant performance is poor. The frame combines the advantages of two materials fully. The axial tensile stress and impact load that is caused by the sample fracture are borne by steel structure during the experiments. The concrete is mainly under positive pressure and used for enhance the longitudinal stiffness of the frame.

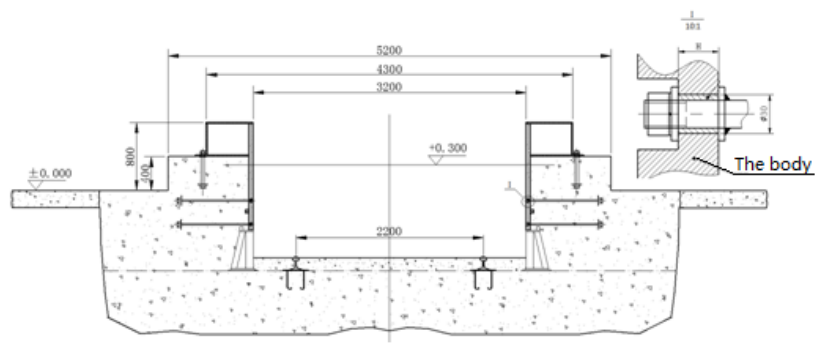

Figure 2. Structure of the frame

\section{Analysis and optimization of the stucture}

\subsection{The grid and its properties}

Simplify the model in some details or the places do not affect the overall stress, and retain the actual structure of the calculation according to the need as much as possible 4. Then import the simplified PRO/E model into Hypermesh and using hexahedral grid to the model after geometry cleanup. Use C3D8I unit type to get 82445 unit numbers and 89495 node numbers. The finite element model is set up as shown in figure 3. Set the material of the frame to Q345B, ultimate strength equal to $510 \mathrm{MPa}$, yield limit equal to $345 \mathrm{MPa}$, modulus of elasticity yield limit 2.12 e5 $\mathrm{MPa}$, poisson ratio equal to 0.31 and the density equal to $7.85 \mathrm{~g} / \mathrm{cm} 3$. 


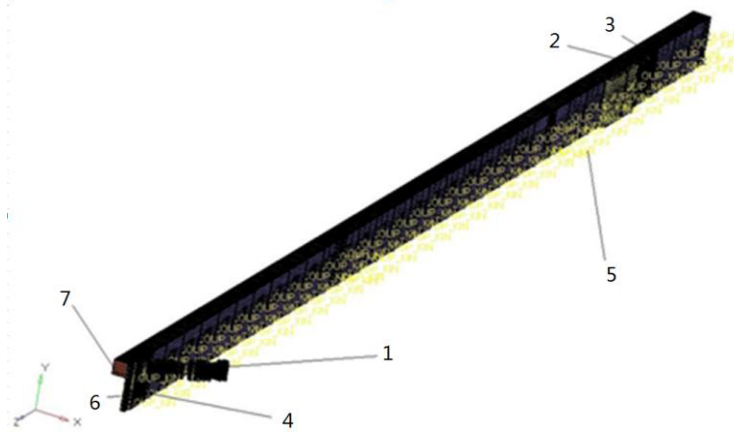

1 - Beam of hydraulic cylinder 2 - work beam, 3 - pin hole, 4 - slotted hole, 5 - concrete, 6 - the frame, 7 baffle

Figure 3. Finite element model

\subsection{Load constraint handling}

Combining with the figure 4 to apply boundary conditions of the model relative to the global coordinate system.

a. add restrictions: 1 . The support in $\mathrm{x}$ direction of hydraulic cylinder beam for the frame and $\mathrm{x}$ direction is fixed;2. The support in $\mathrm{x}$ direction of working beam for the frame and $\mathrm{x}$ direction is fixed; 3. Simulate the connection of anchor bolt and slotted hole, couple slotted hole and limit coupled point in $\mathrm{x}$, y direction; 4 . The support in $\mathrm{x}$ direction of concrete for the frame and $\mathrm{x}$ direction is fixed; 5. The support in y direction of concrete for the frame and y direction is fixed;6. Baffle boundary: $y$ direction is fixed.

b. apply load: the size of connected part between working beam and the frame is about $400 \mathrm{~mm} * 120 \mathrm{~mm}$, the maximum force that working beam gives on the frame is $6000 \mathrm{KN}$, apply the force in the analysis of $6000 /(400 * 120)=125 \mathrm{MPa}$.

\subsection{The results of the analysis}

Figure 4 shows the analysis nephogram of reactive force in the place which stand the force of the frame. The maximum reactive force is $665.1 \mathrm{KN}$, appearing in the place of the third bolt near the origin of force. The value of reactive force is too big, so the structure needs to be modified to improve the force of the whole distribution.

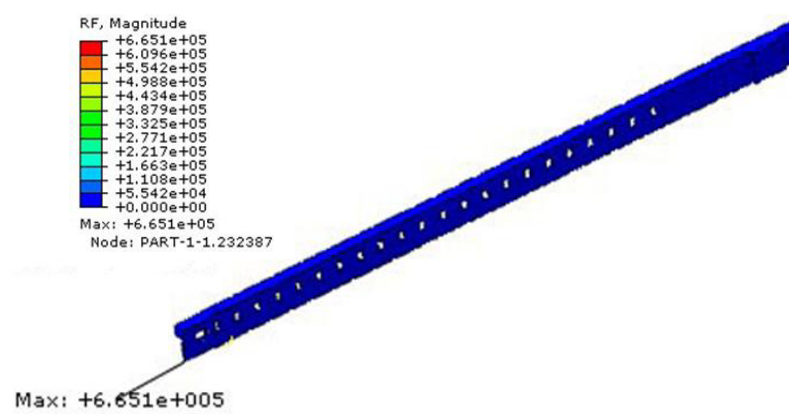

Figure 4. Analysis nephogram of reactive force 1
It exists large defect of reactive force on the local scale by the analysis. In order to reduce the force of the anchor bolts, the measure is that welding unequal angle steel under the steel plate. The model is shown in figure 5 . Then analyze the improved model. The process of parameter setting is the same as above. The results of analysis are as follows.

Figure 6 is the analysis nephogram of reactive force of the improved structure. It can be concluded that the biggest reactive force is about $330 \mathrm{KN}$ which reduces nearly half than the previous structure, so the improved structure is effective. Figure 7 is the stress distribution nephogram of the frame. The maximum stress is 263 $\mathrm{MPa}$, appearing in the place of applied load. The maximum stress is less than $345 \mathrm{MPa}$ of the material yield limit point. Figure 8 is overall displacement nephogram of the frame, the maximum displacement is $0.5 \mathrm{~mm}$ in the position of the applied load, and the deformation is small complying with the design requirements.

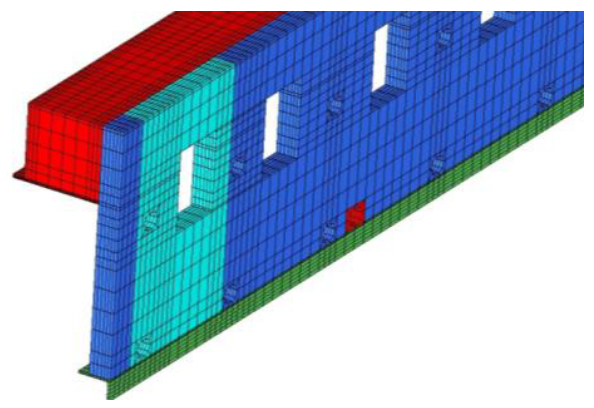

Figure 5. The improved model

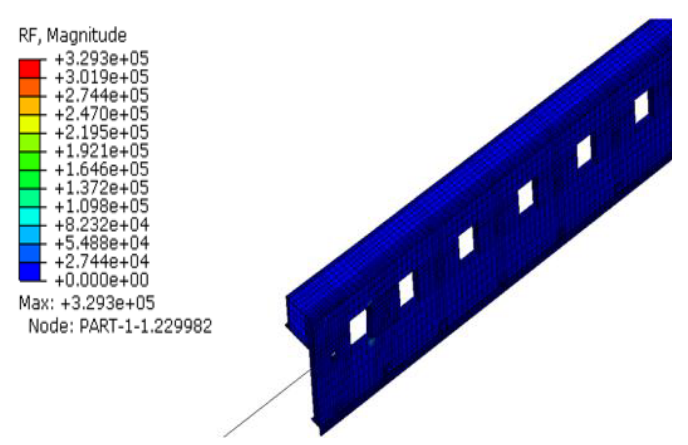

Figure 6. Analysis nephogram of reactive force 2

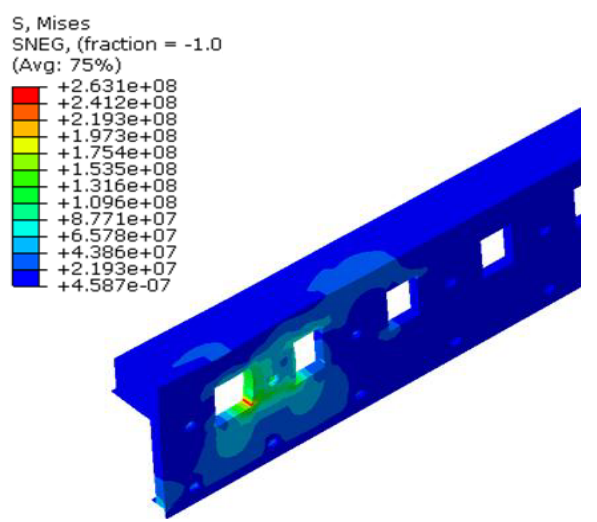

Figure 7. Stress distribution nephogram

\subsection{Structure optimization}




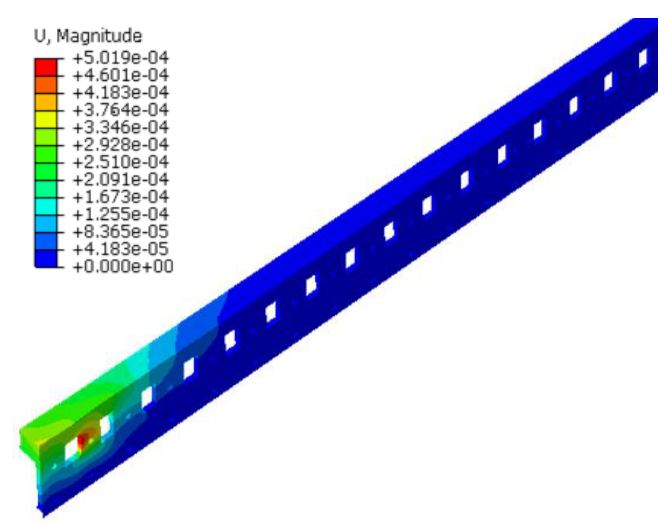

Figure 8. Displacement nephogram

\subsection{Stability analysis of structure}

A reliable frame not only need to meet the design requirements of strength, but also have enough stiffness to ensure performance. Therefore, it is necessary to carry out buckling analysis on the frame to investigate its stability. Using discrete shell element model to the frame and the kinetic coupled unit simulation at the joint of the frame and beam (figure 9), one end fixes and another end loads $12000 \mathrm{KN}$ tension5. Known from the buckling analysis (figure 10), the frame at load of $17760 \mathrm{KN}$ loses instability, so it won't lose instability at load of $12000 \mathrm{KN}$.

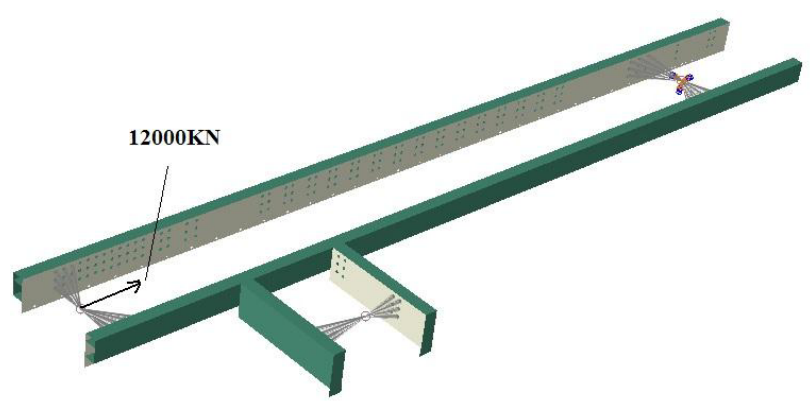

Figure 9. The coupled model of the frame and beam

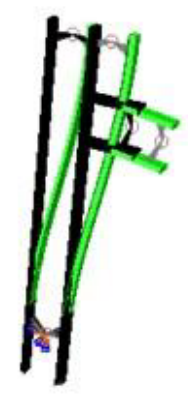

Figure 10. The result of buckling analysis

\section{Application of the frame}

The platform is installed and debugged in Zhoushan Institute of Calibration and Testing for Quality and Technology Supervision successfully, measured by National Institute of Meti-ology. The result is that all aspects of performance meet the national first-class standard. the complete platform is shown in figure 11 .

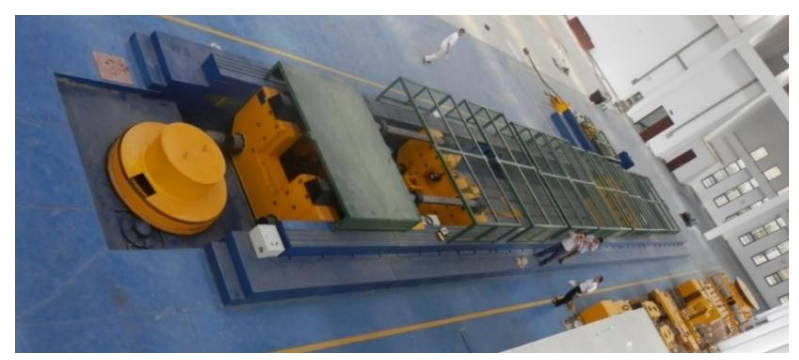

Figure 11. The complete platform

\section{Conclusion}

Traditional test platform frame structure has mature technology, but the weight of the structure is too big and easy to form larger stress in the place of bolt. The paper applies the composite structure of steel and concrete to large test platform. The structure make full use of the advantages that good elasticity and toughness, high stiffness, good compressive performance and low cost. The finite element analysis shows that the initial design of the frame has the disadvantage of too large reactive force on the local scale. After welding unequal angle steel under the steel plate, the stress of the anchor bolts are reduced and reactive force reduces nearly half successfully. Other results of analysis follow up with the design requirements, and also shows that the improved frame is more reasonable.

\section{References}

1. Zhong Xiao-ling, Lu ya-xiong, "The Design of the Emergency Towing Device for YK Oil Tanker," JOURNAL OF SSSRI. 21(1):54-59 (1998).

2. LI Peng, Liu Cong-rui, " Application of a variety of structural frameworks in tension and compression testing machine," Internet of Things. 11:51-53 (2013).

3. Tao Bi-guo, " Design Method and Its Optimization Measures of Concrete Structure," Urbanism and Architecture. Feb 2014:37 (2014).

4. Li Chao, Wang Su-zhen, " Finite Element Analysis on the Loading Frame of Large-type Compression Testing Machine," ENGINEERING \& TEST. Dec 2011:31-33 (2011).

5. Wei Jing, Sun Qing-chao, Sun Wei, Zhao Fei, Li Yong-hong, Guo Ai-gui, " Dynamical coupling characteristics of a large wind turbine gearbox transmission system," JOURNAL OF VIBRATION AND SHOCK. 31(8):16-23 (2012). 